\title{
On Dehn's Algorithm
}

\author{
ROGER C. LYNDON*
}

\section{Introduction}

The Word Problem for groups was formulated by DEHN in 1912, who gave a solution for the fundamental groups of two dimensional manifolds. DEHN's method was geometrical. (See DeHN [5,6], also REIDEMEISTER [17].) Related results have since been obtained by other methods, in particular by TARTAKOVSKII [20-24], SCHIEK [18], BRITTON [2, 3], and GREENDLINGER [9-12]. Here we return to DEHN's geometrical method, extending it to obtain most of these more recent results, together with some results that are new.

DEHN's method is based on the Cayley color diagram, or Gruppenbild, $M$, associated with a presentation of a group $G$. This is a certain abstract two dimensional complex (or, what comes to the same in the present context, the one skeleton of the complex). Let $G=F / N$, with $F$ free on a basis of generators $x_{1}, x_{2}, \ldots$, and $N$ the normal closure in $F$ of a set $R$ of relators $r_{1}, r_{2}, \ldots$ The group diagram $M$ is defined as follows. It has vertices $P(g)$ in one to one correspondence with the elements $g$ of $G$. For each $g$ in $G$ and each $y=x_{i}^{ \pm 1}$, it has an oriented edge $E$ leading from $P(g)$ to $P(g \vec{y})$, where $\bar{y}=y N$; to $E$ is attached the label $\phi(E)=y$, and to the oppositely oriented edge $E^{-1}$ the label $\phi\left(E^{-1}\right)=y^{-1}$. The two cells $D$ of $M$ are as follows: for each closed path $W$ in $M$, such that the labels on the successive edges of $W$ are the successive letters of the reduced word for some $r_{i}$ in $R$, we introduce a two cell $D$ with boundary $W$.

The words $w$ in the $x_{i}^{+1}$ correspond one to one to the paths $W$ in $M$ with base point $P(1)=0$, and $w$ represents an element of $N$ iff $W$ is a closed path. If $w$ is moreover reduced, then $W$ is spanned by a possibly singular union of two cells $D$, together with spines attaching them to the base point 0 . In the cases considered by DEHN, the group diagram $M$ can be embedded in the plane. Then, if $w$ is the reduced word for a non trivial element of $N$, the path $W$ will contain a simple closed subpath $W_{0}$ at some point $P_{0}$, and $W_{0}$ will be the boundary $U$, at $P_{0}$, of a simple finite union $U$ of two cells $D$ in the plane, each corresponding to some relator $r_{i}$ or its inverse $r_{i}^{-1}$.

Now restrictions on the amount that two reduced words $r_{i}^{ \pm 1}$ can have in common provide conditions on the amount of common boundary between two adjoining discs $D$ in $U$. At this point DeHN used the fact that (apart from trivial cases) $M$ can be represented as a regular tesselation of the hyperbolic plane with Poincare metric, and inferred on metric grounds that the boundary $W_{0}$ of $U$ must contain more than half the boundary $D^{*}$ of some disc $D$. (REIDE-

* I wish to acknowledge the hospitality of Queen Mary College, London, where this work was done, and also the support of the National Science Foundation. 
MEISTER [17] later showed that this conclusion can be obtained purely combinatorially, without appeal to a metric.) It follows immediately that the reduced word $w$ must contain more than half of some cyclic permutation of the reduced word for some $r_{i}^{ \pm 1}$.

The Word Problem asks whether arbitrary $w$ in $F$ belongs in fact to $N$. If $w=1$, then $w$ is in $N$. Otherwise, given a non trivial reduced word $w$ in $F$, it can be decided by inspection whether $w$ contains more than half of some cyclically reduced conjugate $s$ of an $r_{i}^{ \pm 1}$. If not, then, by the above, $w$ is not in $N$. If so, say, $w=a b c$ with $s=b d$, and $d$ shorter than $b$, then $w$ is in $N$ iff the shorter word $w^{\prime}=a d^{-1} c$ is in $N$. A finite number of such reductions then leads to a decision whether $w$ is in $N$.

We depart from DeHN's method mainly in not requiring that the entire group diagram $M$ be embeddable in the plane. For quite general $G=F / N$, and arbitrary $w$ in $N$, there exists a finite complex $M^{\prime}$ in the plane, with boundary described by a closed path on which the product of the labels is $w$, and with interior a union of discs corresponding to relators, in exactly the same way as in DeHN's case, where all of $M$ can simultaneously be embedded in the plane. Conditions on the amount of agreement between relators now lead as before to conditions on common boundaries between discs, and to a solution of the Word Problem. Indeed, by a modified combinatorial argument, we are able to solve the Word Problem in additional cases where non trivial $w$ in $N$ need not contain as much as half of any cyclically reduced conjugate of a relator.

In addition to the Word Problem, the present methods yield a proof that, in the cases treated, the commutator quotient group $N /[N, N]$ of $N$, viewed as a natural $G=F / N$ module, if not free, is at least a direct sum of cyclic submodules, whence the cohomology of $G$, beginning with dimension 3 , has period 2 .

The present methods, with minor modification, apply equally to the case that $F$ is an arbitrary free product, rather than a free group. This case, already considered by SCHIEK [18] and BRITTON [3], has bearing on the problem of adjunction of solutions to equations over a group.

The main argument falls naturally into three stages. In Section 2 we discuss finite complexes (graphs, or maps) in the plane. In Section 3, we associate plane diagrams with elements of a free group. In Section 4, this connection is used to translate properties of plane graphs into properties of groups: this contains our main results, Theorems I, II, and III. A further Section 5 deals with free products, with examples illustrating applications to the Adjunction Problem.

In addition to references cited explicitly, I want to acknowledge suggestions from B. J. Birch, D. E. COHEN, H. SCHIEK, and H. Zieschang.

\section{Maps}

A map $M$ in the plane $\Pi$ is determined by a finite set of points, called vertices, and of simple arcs, called edges. Both ends of an edge are vertices (not necessarily distinct), and an edge is not incident elsewhere with any vertex or any other edge. A region $D$ of a map $M$ is a bounded component of the complement $I-M$ 
of $M$ in the plane $\Pi$; we consider only maps in which every region is simply connected.

The degree $d(P)$ of a vertex $P$, in $M$, is the number of edges incident at $P$, any edge with both ends at $P$ being counted twice. The degree $d(D)$ of a region $D$ is the number of edges on the boundary $D^{\circ}$ of $D$, any edge such that $D$ lies on both sides of it being counted twice.

The boundary $M^{*}$ of $M$ is the topological boundary of the unbounded component of the complement $\Pi-M$. A boundary vertex or edge is one contained in $M^{*}$, and a boundary region $D$ is one whose boundary $D^{*}$ contains a boundary edge. Those not on the boundary are interior vertices, edges, and regions.

Our main results about maps can be viewed as combinatorial versions of two elementary metric properties of sufficiently regular domains in the Euclidean plane. The first is that the integral of curvature along the boundary $M^{*}$ of $M$ is $2 \pi$, and the second that the area of $M$ does not exceed a constant $(1 / 4 \pi)$ times the square of the length of the boundary $M$. To introduce the ideas, we suppose that $M$ is a union of regions in a regular tesselation $T$ of the Euclidean plane. There are, of course, three types of regular tesselation, by hexagons, by squares, and by triangles, and our later results fall into three corresponding cases; but, for the precent heuristic remarks, we confine attention to a regular tesselation $T$ by hexagons. We suppose, for the moment, that the vertices, edges, and regions of the map $M$ belong to the regular tesselation $T$, and that $M$ is a simple closed polygon, containing more than one hexagon from $T$.

To calculate curvature along $M$, we pass to the closed polygon $C$ (in the tesselation $T^{*}$ dual to $T$ ) whose successive vertices are the centers $D^{*}$ of the successive regions $D$ lying on the boundary $M$ of $M$. It is easy to see that, if $i(D)$ is the number of edges on $D^{\prime}$ that are interior to $M$, then the change of direction in $C$ at the point $D^{*}$ is $[4-i(D)] \frac{2 \pi}{6}$. It follows that

$$
\Sigma[4-i(D)] \frac{2 \pi}{6}=2 \pi,
$$

where the notation indicates summation over all boundary regions $D$ of $M$, and hence that

$$
\Sigma[4-i(D)]=6 .
$$

In the map $M$, contained in $T$, we evidently have $d(D)=6$ for each of the hexagonal regions $D$ of $M$, and $d(P)=3$ for every interior vertex $P$ of $M$. We now pass to more general maps $M$, requiring only that $M$ contain more than one region, that $d(D) \geqq 6$ for every region $D$ of $M$, and that $d(P) \geqq 3$ for every interior vertex $P$ of $M$. We shall see that, in this case, the equation above may be replaced by an inequality,

$$
\Sigma[4-i(D)] \geqq 6 \text {. }
$$

An alternative formulation of this result refers to a map $M^{*} d u a l$ to $M$. The combinatorial relation of $M^{*}$ to $M$ is determined uniquely by the condition that $M^{*}$ has exactly one vertex $D^{*}$ within each region $D$ of $M$, and one edge $E^{*}$ corresponding to each interior edge $E$ of $M$, crossing $E$ to join the vertices $D_{1}^{*}$ and $D_{2}^{*}$ of $M^{*}$ contained in the regions $D_{1}$ and $D_{2}$ of $M$ abutting along $E$. Note 
that $M^{* *}$ is not isomorphic to $M$, but can be identified with the submap of $M$ obtained by deleting all vertices on $M^{*}$ and all edges incident with such a vertex. Replacing $M$ by $M^{*}$, the above hypotheses require that $M$ contain more than one vertex, that $d(D) \geqq 3$ for all $D$, and that $d(P) \geqq 6$ for all interior vertices $P$. The conclusion then takes the form that

$$
\Sigma[4-d(P)] \geqq 6 .
$$

One immediate consequence of this is that, under the hypotheses, $M$. contains at least two convex points $P$, that is, with $d(P) \leqq 3$. A second is that there exists no map $M$ on the sphere, in the obvious sense, with all $d(D) \geqq 6$ and all $d(P) \geqq 3$.

To relate area and length of boundary, we again suppose for the moment that $M$ is part of the regular tesselation $T$. Then the area of $M$ is proportionate to the number $a(M)$ of regions $D$ of $M$, and the length of $M$ to the number of edges on $M$, that is, to $b(M)=\Sigma[6-i(D)]$. In this case it is obvious that $a(M)$ is bounded by a constant multiple of $b(M)^{2}$. It will be seen that for more general $M$, such that $d(D) \geqq 6$ for all $D$ and that $d(P) \geqq 3$ for all interior $P$, we have indeed

$$
a(M) \leqq \frac{1}{36} b(M)^{2} \text {. }
$$

We turn now to exact statements and proofs of these results. We begin with a rather general formula; the present method of proof was suggested by BiRCH. If $M$ is any map in the plane, we write $Q$ for the number of connected components of $M$, and $Q_{0}$ for the number that consist of a single isolated point. We define a generalization of the expression found above for curvature, depending on a real parameter $t$, by the formula

$$
K(t)=\Sigma[t-d(P)],
$$

where summation is over all $P$ on the boundary $M$ of $M$. As a measure of the excess of degree over $t$, on all interior vertices, we take

$$
V(t)=\Sigma^{0}[d(P)-t]
$$

sum over all interior vertices, $P$ on $M-M$. As a measure of excess degree of regions we take

$$
R(t)=\Sigma[d(D)-t],
$$

sum over all regions $D$ of $M$. In these terms we state our first basic formula.

Lemma 2.1. Let $M$ be any map, and $p$ and $q$ real numbers such that $1 / p+1 / q$ $=1 / 2$. Then

$$
K\left(\frac{p}{q}+2\right) \geqq p Q+V(p)+\frac{p}{q} R(q)-\frac{p}{q} Q_{0} .
$$

We begin the proof with EULER's formula, in the form

$$
Q=v-e+d \text {, }
$$

where $v, e$, and $d$ are the numbers of vertices, edges, and regions. It is immediate that

(3) $2 e=\Sigma d(D)+d_{\infty}$, sum over all $D$ in $M$, with $d_{\infty}$ the number of edges $d\left(D_{\infty}\right)$ on the unbounded component of $\Pi-M$. 
To eliminate $e$ from these three equations we combine (2) multiplied by a real number $m$, and (3) multiplied by a real number $n$, with (1) multiplied by $2(m+n)$. Putting aside the cases that $m=0, n=0$, or $m+n=0$, we may normalize, taking $m=1$ and $n \neq 0,-1$. We obtain then

$$
\begin{aligned}
2(n+1) Q= & 2(n+1) v-\Sigma d(P)+2(n+1) d-n \Sigma d(D)-n d_{\infty} \\
& =\Sigma[2(n+1)-d(P)]+n \Sigma\left[\frac{2(n+1)}{n}-d(D)\right]-n d_{\infty} .
\end{aligned}
$$

Now the real solutions of $1 / p+1 / q=1 / 2$ are given parametrically by $p=2(n+1)$ and $q=2(n+1) / n$, for $n \neq 0,-1$. With these substitutions we have

$$
\begin{aligned}
p Q & =\Sigma[p-d(P)]+\frac{p}{q} \Sigma\left[q-d(D)-n d_{\infty}\right. \\
& =-V(p)+\Sigma[2(n+1)-d(P)]-\frac{p}{q} R(q)-n d_{\infty} .
\end{aligned}
$$

To remove the term in $d_{\infty}$ we note that the number $v_{\infty}$ of vertices on $M$ satisfies the relation

$$
v_{\infty} \leqq d_{\infty}+Q_{0},
$$

since, in traversing $M^{*}$ in the positive sense, each non isolated point of $M^{*}$ is encountered as the beginning of at least one distinct edge on $M$. With this we have

$$
\begin{aligned}
p Q & =-V(p)+\Sigma[n+2-d(P)]-\frac{p}{q} R(q)+n v_{\infty}-n d_{\infty} \leqq \\
& \leqq-V(p)+K\left(\frac{p}{q}+2\right)-\frac{p}{q} R(q)+\frac{p}{q} Q_{0} .
\end{aligned}
$$

This completes the proof.

The essence of this formula is contained in the case that $M$ is a simple closed curve. Here we have $Q=1, Q_{0}=0, v_{\infty}=d_{\infty}$, and obtain an exact equality

$$
K\left(\frac{p}{q}+2\right)=p+V(p)+\frac{p}{q} R(q)
$$

which may be viewed as a combinatorial generalization of the Gauss-Bonnet formula for tesselations of the hyperbolic plane. Suppose further that $d(D)>6$ for all $D$ and $d(P) \geqq 3$ for all interior $P$. Taking $p=3$ and $q=6$ we have

$$
K\left(\frac{p}{q}+2\right)=\Sigma\left[\frac{5}{2}-d(P)\right] \leqq \Sigma\left[\frac{5}{2}-2\right]=\frac{1}{2} b(M),
$$

where $b(M)$ is the number of edges on $M$. We have $V(p)=\Sigma^{0}[d(P)-3] \geqq 0$, and

$$
\frac{p}{q} R(q)=\frac{1}{2} \Sigma[d(D)-6] \geqq \frac{1}{2} \Sigma[7-6]=\frac{1}{2} a(M),
$$

where $a(M)$ is the number of regions of $M$. From this we have $a(M) \leqq b(M)-6$, a linear relation between area and length of boundary, which can also be inferred from DEHN's algorithm for this case. 
We comment also that the proof of Lemma 2.1 indicates how the condition $1 / p+1 / q=1 / 2$ enters into our considerations, independent of any metric, Euclidean or otherwise. In the sequel, we shall always take $p$ and $q$ to be positive integers, hence $(p, q)$ one of the pairs $(6,3),(4,4)$, and $(3,6)$, corresponding to the three Euclidean tesselations.

Corollary 2.2. Let $(p, q)=(6,3),(4,4)$, or $(3,6)$. Let $M$ be a map containing more than one vertex, and such that $d(D) \geqq q$ for every region $D$ and that $d(P) \geqq p$ for every interior vertex $P$. Then, according to the three cases,

$$
\Sigma[4-d(P)] \geqq 6, \quad \Sigma[3-d(P)] \geqq 4, \quad \text { or } \quad \Sigma\left[\frac{5}{2}-d(P)\right] \geqq 3 .
$$

Corollary 2.3. Under the same hypotheses, the boundary $M$ of $M$ contains at least two points $P$ such that, according to the case, $d(P) \leqq 3, d(P) \leqq 2$, or $d(P) \leqq 2$.

Corollary 2.4. Let $(p, q)=(6,3),(4,4)$, or $(3,6)$. Let $M$ be a map containing more than one region, and such that $d(D) \geqq p$ for each interior region $D$ and that $d(P) \geqq q$ for each interior vertex $P$. Then there are at least two regions $D$ on the boundary $M$ of $M$ such that, according to the case, $D^{\prime}$ contains no more than 3 , 2 , or 2 interior edges.

Corollary 2.2 is an immediate consequence of Lemma 2.1, and Corollary 2.3 is an immediate consequence of Corollary 2.2 . To prove Corollary 2.4 , let $M$ satisfy the given hypotheses, and let $M^{*}$ be a map dual to $M$. Since $M$ contains more that one region $D, M^{*}$ contains at least two corresponding vertices $D^{*}$. Each region of $M^{*}$ contains some interior vertex $P$ of $M$, and has the same degree, $d(P) \geqq q$. Each interior vertex of $M^{*}$ is the $D^{*}$ contained in some interior region $D$ of $M$, and has the same degree, $d(D) \geqq p$. Thus $M^{*}$ satisfies the hypotheses, and so the conclusion, of Corollary 2.3. Finally, a vertex $D^{*}$ of $M^{*}$ with $d\left(D^{*}\right) \leqq 3$, 2 , or 2, is contained in a region $D$ of $M$ with no more than 3,2 , or 2 interior edges.

We note that the case $(p, q)=(4,4)$ of Corollary 2.4 is closely related to a theorem of REIDEMEISTER [17], page 201.

A map on the two dimensional sphere is defined exactly as a map on the plane, except that now every component of the complement is counted as a region.

Corollary 2.5. Let $(p, q)=(6,3),(4,4)$, or $(3,6)$. Then there does not exist any map $M$ on the sphere such that $d(D) \geqq p$ for all regions $D$ and that $d(P) \geqq q$ for all vertices $P$.

To see this, suppose such a map $M$ on the sphere given. Deleting a point $p_{\infty}$ of the sphere, not on $M$, we obtain a plane, and deleting from $M$ the region $D_{\infty}$ containing $p_{\infty}$ we obtain a map $M_{0}$ on the plane. We may suppose $D_{\infty}$ chosen to have no more than one edge in common with any other region $D$. By Corollary $2.4, M_{0}$ contains a region $D$ with no more than 3,2 , or 2 interior edges in $M_{0}$; then $D$, as a region of $M$, has degree $d(D) \leqq 4,3$, or 3 . In the first two cases this contradicts the hypothesis on $M$. The third case follows from the first by duality : if $M$ satisfied the hypotheses with $(p, q)=(3,6)$, than a dual map $M^{*}$ would satisfy the hypothesis with $(p, q)=(6,3)$. 
Although Corollary 2.5 must surely be long known, the nearest related result we can find is in BERGE [1], page 208.

We turn now to our remaining lemma on maps, relating area to length of boundary.

Lemma 2.6. Let $(p, q)=(6,3),(4,4)$, or $(3,6)$. Let $M$ be a map in the plane such that $d(D) \geqq q$ for each region $D$ and that $d(P) \geqq p$ for each interior vertex $P$. Define

$$
\begin{aligned}
& a(M)=\text { number of vertices of } M, \\
& b(M)=\Sigma[p-d(P)], \text { all } P \text { on } M .
\end{aligned}
$$

Then

$$
a(M) \leqq \frac{1}{K} b(M)^{2}
$$

where, according to the case, $K$ is 36,16 , or 6 .

The proof is by induction on the number of vertices of $M$. In the initial case that $M$ contains only a single point, and hence, by the condition $d(D) \geqq q$, no edges, the relation $K a \leqq b^{2}$ evidently holds provided only that $K \leqq p^{2}$. But, it will be seen, the inductive step requires that $K \leqq 2 p(p-2)$. Now the given number 36,16, or 6 is the smaller of $p^{2}$ and $2 p(p-2)$, according as $p$ is 6,4 , or 3 .

As a preliminary to the inductive step, suppose that $M$ consists of a non empty part $M_{0}$, together with one further vertex $P$, either isolated or attached to $M_{0}$ by a single edge. Then $a=a_{0}+1$, with $a_{0} \geqq 1$, while either $b=b_{0}+p$ or $b=b_{0}+p-2$. Now, assuming inductively that $K a_{0} \leqq b_{0}^{2}$, and using the fact that $K \leqq 2 p(p-2)$ and that $b_{0}$ is an integer, it is easy to deduce that $K a=K a_{0}+$ $+K \leqq\left(b_{0}+p-2\right)^{2} \leqq b^{2}$. With this, we may assume henceforth that $M$ contains more than one vertex, and that every vertex on $M^{*}$ is incident with at least two edges on $M$.

Now Corollary 2.2 applies to give $\Sigma\left[\frac{p+2}{2}-d(P)\right]>p$. If we write $a$ for the number of vertices on $M$ and $d=\Sigma d(P)$, this inequality takes the form

$$
\frac{p+2}{2} a-d \geqq p \text {. }
$$

Since $b=\Sigma[p-d(P)]=p a-d$, form (1) we have

$$
b \geqq \frac{p-2}{2} a+p \text {. }
$$

Let $M_{1}=M^{* *}$, the map obtained from $M$ by deleting all vertices on $M$, together with the set $F$ of all edges with one or both ends on $M$. Let $f_{1}$ be the number of such edges with one end on $M$, and $f_{2}$ of those with both ends on $M$. Evidently

$$
d=f_{1}+2 f_{2} \text {. }
$$

Since every vertex on $M$ is incident with at least two edges on $M$, we have $2 a \leqq 2 f_{2}$, that is,

$$
a \leqq f_{2}
$$


We now estimate $b_{1}=b\left(M_{1}\right)=\Sigma_{1}\left[p-d_{1}(P)\right]$, where the sum is over all $P$ on $M_{1}$ and where $d_{1}(P)$ is the degree of $P$ in $M_{1}$. First, if $P$ is in $M_{1}$, then $P$ is not on $M$, whence $d(P) \geqq p$. Second, from the definition of $M_{1}$, if $P$ is in $M_{1}$, then $d_{1}(P)$ $=d(P)-f(P)$, where $f(P)$ is the number of edges of the set $F$ incident at $P$. Evidently $\Sigma_{1} f(P)=f_{1}$. Now

$$
b_{1}=\Sigma_{1}\left[p-d_{1}(P)\right] \leqq \Sigma_{1}\left[d(P)-d_{1}(P)\right]=\Sigma_{1} f(P)=f_{1},
$$

whence

$$
b-b_{1}=p a-d-f_{1}
$$

and using successively (3), (4), and (1), we have

$$
b-b_{1}=p a-2 d+2 f_{2} \geqq(p+2) a-2 d \geqq 2 p,
$$

that is,

$$
b_{1} \leqq b-2 p .
$$

Assume inductively that $K a_{1} \leqq b_{1}^{2}$, where $K \leqq 2 p(p-2)$. Since $a=a+a_{1}$, we have $K a=K a+K a_{1} \leqq 2 p(p-2) a+b_{1}^{2}$, and estimating $a^{\cdot}$ and $b_{1}$ by (2) and (5) gives $K a \leqq 4 p(b-p)+(b-2 p)^{2}=b^{2}$.

\section{Diagrams}

A diagram, over a group $F$, consists of a finite map $M$ equipped with a function $\phi$ assigning to each oriented edge $E$ as label a non trivial element $\phi(E)$ of $F$, and assigning the inverse element to the oppositely oriented edge $E^{-1}$. We shall associate a diagram $M$ with each finite sequence $p_{1}, \ldots, p_{n}$ of non trivial elements from $F$, a free group with given basis. Note that each $p_{i}$ has a unique representation $p_{i}=u_{i} r_{i} u_{i}^{-1}$ in reduced form, where $r_{i}$ is cyclically reduced.

A diagram $M$ will be called a diagram for the sequence $p_{1}, \ldots, p_{n}$ if it satisfies the following two conditions.

(1) The boundary $M^{\top}$ is connected and contains a vertex 0 such that, if $E_{1}, \ldots, E_{k}$ are the successive oriented edges traversed in running around $\boldsymbol{M}$ from 0 back to 0 in the positive sense, then the product of the labels $\phi\left(E_{1}\right), \ldots$, $\phi\left(E_{k}\right)$ is reduced without cancellation, and is the reduced word for $w=p_{1} \ldots p_{n}$.

(2) If $D$ is any region of $M$, then its boundary $D^{\circ}$ contains a point $P$ such that, if $E_{1}, \ldots, E_{\mathrm{k}}$ are the successive oriented edges traversed in running around $D^{\circ}$ from $P$ back to $P$ in the positive sense, then the product of the labels $\phi\left(E_{1}\right), \ldots$, $\phi\left(E_{k}\right)$ is reduced without cancellation, and is the reduced word for one of $r_{1}, \ldots, r_{n}$.

Lemma 3.1. There exists a diagram for every sequence.

We shall construct, for each sequence, a canonical diagram.

If $n=0$, and the sequence is empty, we take $M$ to consist of a single point 0 .

Let $n=1$, and $w=p_{1}=u_{1} r_{1} u_{1}^{-1}$. We draw a loop, consisting of a single edge $E$ with both ends at the same vertex $P$, and label $E$, in the positive sense, with $r_{1}$. If $u_{1}=1$, we take $0=P$ and are done. If $u_{1} \neq 1$, we connect an external point 0 to $P$ with an edge $E^{\prime}$, with label $\phi\left(E^{\prime}\right)=u_{1}$. 
Let $n>1$. As in the case $n=1$, we construct diagrams $M_{1}, \ldots, M_{n}$ for the single factors $p_{1}, \ldots, p_{n}$. We may take these with common base point 0 , each external to the rest, and arranged in order about the point 0 . If $M$ is the resulting diagram, then (2) is already satisfied. Moreover, $M^{\prime}$ is connected and contains 0 , and the product of the labels on $M$ from 0 back to 0 (beginning with the first edge of $M_{1}$ ) is equal to $w=p_{1} \ldots p_{n}$. However, this product need not be reduced without cancellation. We shall show how to modify a diagram $M$, satisfying the conditions just stated, until ultimately (1) is satisfied.

For simplicity, if an edge $E$ bears a label with reduced form $v=y_{1}, \ldots, y_{k}$, each $y_{i}$ a generator or the inverse of a generator, we divide $E$ into successive edges $E_{1}, \ldots, E_{k}$ with labels $y_{1}, \ldots, y_{k}$. Thus we may suppose each label is a generator or the inverse of a generator. Let $\alpha$ be an arc, from 0 to 0 , describing $M$, and such that the product of the labels on the successive edges of $\alpha$ is $w$. If (1) is not already satisfied, then $\alpha$ will have the form $\alpha=\beta E_{1} E_{2} \gamma$, where $E_{1}$ and $E_{2}$ are consecutive edges bearing inverse labels. Let $E_{1}$ run from $P_{1}$ to $P_{0}$ and $E_{2}$ from $P_{0}$ to $P_{2}$. Suppose first that $P_{1}$ is distinct from $P_{0}$ and $P_{2}$. Then, deforming that part of $M$ lying in a small neighborhood of $E_{1}$, and keeping $P_{0}$ fixed, we can pivot $E_{1}=P_{1} P_{0}$ about $P_{0}$ in the exterior of $M$ until it comes into coincidence with $E_{2}=P_{2} P_{0}$. Then $P_{1}$ and $P_{2}$ coincide, as do the oriented edges $E_{1}$ and $E_{2}^{-1}$. In resulting $M^{\prime}$ the arc $\alpha$ will be replaced by $\alpha^{\prime}=\beta \gamma$, containing fewer edges than $\alpha$. If $P_{2}$ is distinct from $P_{0}$ and $P_{1}$, we proceed similarly. The case remains that $P_{1}=P_{2}$. In this case the part $E_{1} E^{2}$ of $\alpha$ is a loop, attached to the rest of $\alpha$ only at the point $P_{0}$. We form $M^{\prime}$ by deleting this loop, together with all of $M$ interior to the loop; again $\alpha$ is replaced by $\alpha^{\prime}=\beta \gamma$. In all cases, $M^{\prime}$ satisfies the same hypotheses as $M$, whence, by iteration, we must ultimately arrive at some $M^{\prime \prime}$ satisfying (1) as well as (2).

Suppose that, in obtaining the reduced form for the product $w=p_{1} \ldots p_{n}$, there is cancellation between corresponding parts of some $p_{i}$ and $p_{j}$, for $1 \leqq i<j \leqq n$, in the following sense. First, $r_{i}$ and $r_{j}$ have reduced forms $r_{i}=a x b$ and $r_{j}=c x^{-1} d$, where the parts $x$ and $x^{-1}$ correspond in the sense that cyclic conjugates $x b a$ and $x c^{-1} d^{-1}$ of $r_{i}$ and $r_{j}^{-1}$ are equal, hence $b a=c^{-1} d^{-1}$. Second, the corresponding parts cancel, that is, $x b u_{i}^{-1} q u_{j} c x^{-1}=1$ where $q=p_{i+1} \ldots p_{j-1}$. Then we have $p_{i} q p_{j}=u_{i} a d u_{j}^{-1}$, and since $a d=b^{-1} c^{-1}$ and $q=u_{i} b^{-1} c^{-1} u_{j}^{-1}$, we have $p_{i} q p_{j}=q$. It follows that $w=p_{1} \ldots p_{i-1} p_{i+1} \ldots p_{j-1} \times$ $\times p_{j+1} \ldots p_{n}$.

We shall call a sequence $p_{1}, \ldots, p_{n}$ reduced if, for no $i$ and $j$, where $1 \leqq i<$ $<j \leqq n$, do we have $p_{i} \ldots p_{j}=p_{i+1} \ldots p_{j-1}$. Then if $w$ is the product of a sequence, it is the product of some reduced subsequence.

We note that reduced sequences have been considered by GREENDLINGER [9], page 68, and by GLADKII [8].

A diagram $M$ will be called reduced if it contains no interior edge $E$, separating regions $D_{1}$ and $D_{2}$, such that the product of the labels on the boundary $D^{\circ}$ of the combined region $D=D_{1} \cup D_{2} \cup E$ reduces to 1 . Suppose the canonical diagram $M$, constructed above, for a sequence $p_{1}, \ldots, p_{n}$ is not reduced. For $E, D_{1}$ and $D_{2}$ as above we have $D_{1}$ and $D_{2}^{*}$ described by $\operatorname{arcs} E \delta_{1}$ and 
$E^{-1} \delta_{2}$, where the product of the labels on the arc $\delta_{1} \delta_{2}$ describing $D^{\circ}$ is 1 . Now $E$ must have entered $M$ through identification of edges $E_{1}$ from a certain $M_{i}$ and $E_{2}^{-1}$ from a certain $M_{j}$, where we may suppose $i<j$. Thus $M_{i}$ contains $D_{1}$ with $D_{1}$ described by $E_{1} \delta_{1}$ where $r_{i}=a x b$, for $\phi\left(E_{1}\right)=x$, and $b a$ the product of the labels on $\delta_{1}$. Similarly, $r_{j} \equiv c x^{-1} d$, with $d c$ the product of the labels on $\delta_{2}$. The product of the labels $\delta_{1} \delta_{2}$ is then bade $=1$. Moreover, in order that $E_{1}$ and $E_{2}$ should become successive edges, the product of the intervening edges on $M_{1} \cup \cdots \cup M_{n}$ must have reduced to 1 , that is, $b u_{i}^{-1} p_{i+1} \ldots p_{j-1} u_{j} c=1$. But this implies that the sequence $p_{1}, \ldots, p_{n}$ is not reduced. We have proved the following.

Lemma 3.2. Every reduced sequence possesses a reduced diagram.

In fact, if $p_{1}, \ldots, p_{n}$ is any sequence, it contains a reduced subsequence with the same product $w$, and any reduced diagram for the subsequence will be a reduced diagram for the original sequence. One can, alternatively, obtained a reduced diagram for any sequence by successively shrinking to a slit any combined region $D$ as above with the product of the labels on $D^{\circ}$ equal to 1 . This can be done as well in the case that $D$ is the union of more than two regions, together with their separating edges.

We next examine the possibility of the loss of some of the $n$ regions of $M_{1} \cup \cdots \cup M_{n}$ in the course of constructing the canonical diagram $M$ for a reduced sequence $p_{1}, \ldots, p_{n}$. This can happen only in case, in the construction of $M$, we encounter a loop $E_{1} E_{2}$ on the boundary. Let $L_{0}$ be the part of the diagram consisting of this loop and everything interior to it. Completing the plane to a sphere by adjoining a point $p_{\infty}$, we can flatten the new region $D_{\infty}$ containing $p_{\infty}$ to a slit, bringing the two edges $E_{1}$ and $E_{2}^{-1}$ into coincidence. We then have a diagram $L$ on the sphere which satisfies (2). Now $L$ will be a reduced diagram unless identification of $E_{1}$ and $E_{2}^{-1}$ violates the condition for a reduced diagram. But then exactly the same argument as before would show that the labels $x$ on $E_{1}$ and $x^{-1}$ on $E_{2}$ were corresponding parts of certain $r_{i}$ and $r_{j}$, which could be cancelled in reducing the product $p_{1} \ldots p_{n}$. This would contradict the assumption that $p_{1}, \ldots, p_{n}$ is reduced. This proves the following.

Lemma 3.3. If $p_{1}, \ldots, p_{n}$ is a reduced sequence, then there exists a reduced diagram $M$ for this sequence, together with some number $q \geqq 0$ of reduced diagrams $L_{1}, \ldots, L_{q}$ on spheres, such that these diagrams contain in all exactly $n$ regions.

A subdiagram $M_{0}$ of a reduced diagram $M$ for $p_{1}, \ldots, p_{n}$ will be called critical if either (i) $M_{0}$ contains a single region $D$, and a closed arc bounding $D$ is part of a closed arc at 0 bounding $M$, or else if (ii) $M_{0}$ contains more than one region, and with the possible exception of one region $D_{0}$, for every $D$ in $M_{0}$, such that $D \cap M_{0}$ contains more than a single point, $D \cap M_{0}$ is a simple arc and a part of a closed arc at 0 bounding $M$.

Lemma 3.4. If $p_{1}, \ldots, p_{n}$ is a reduced sequence, and $p_{1} \ldots p_{n} \neq 1$, then the sequence possesses a critical subdiagram.

Let $M$ be a reduced diagram. From (1) it follows, since $p_{1} \ldots p_{n} \neq 1$, that $M$ consists of more than the single point 0 , and that $M^{\prime}$ contains no spines, that is, successive edges $E$ and $E^{-1}$, except possibly at 0 . It follows that, if $\alpha$ is an arc 
with base point 0 that describes $M$, then $\alpha$ must contain a simple loop $\beta$ at some base point $P$. Let $M_{0}$ be the subdiagram of $M$ consisting of $\beta$ together with all of $M$ contained within $\beta$; then $\beta$ describes $M_{0}$, and $M_{0}$ has at most the point $P$ in common with the remainder of $M$. If $M_{0}$ contains a single region $D$, then $\beta$ describes $D$, and, since $\beta$ is part of $\alpha, M_{0}$ is critical. Suppose $M_{0}$ contains more than one region. Choose a region $D_{0}$ of $M_{0}$ such that $P$ lies on $D_{0}^{\circ}$. If $D$ is a region of $M_{0}$ other than $D_{0}$, and if $D^{\circ} \cap M$ is connected and consists of more than one point, then $D^{\circ} \cap M^{\circ}$ is an arc $\delta$, not containing $P$ in its interior, and hence is a simple subarc of $\beta$ and so of $\alpha$. Thus again $M_{0}$ is critical provided every $D^{\circ} \cap M_{0}$ is connected.

Suppose $D_{1}$ is a region of $M_{0}$ such that $D_{1} \cap M_{0}$ is not connected. Then removing $D_{1}^{\prime}$ separates $M_{0}$ into more than one component, and we may choose a component $K$ not containing $P$. Let $M_{1}=K \cup D_{1}^{\circ}$, and suppose first $D_{1}$ and then $K$ chosen to make the number of regions in $M_{1}$ as small as possible. Then $D^{\prime} \cap M_{1}$ is connected for all $D$ in $M_{1}$. Moreover, $M_{1}$ contains some region other than $D_{1}$, and if $D$ is such a region, and $D^{\prime} \cap M_{1}$ contains more than one point, it is an arc not containing $P$ in its interior, and hence a simple subarc of $\alpha$. Therefore $M_{1}$ is critical. This establishes Lemma 3.4.

It is perhaps worth commenting on the relation between the diagram $M$ associated with a sequence $p_{1}, \ldots, p_{n}$ and the full group diagram $\bar{M}$ for the presentation determined by the relators $r_{1}, \ldots, r_{n}$. An incidence preserving map $\psi$ from $M$ into $\bar{M}$ is determined by mapping the vertex 0 of $M$ into the vertex $P(1)$ of $\bar{M}$, and each edge $E$ of $M$ with starting point $P$ and label $y=x_{i}^{ \pm 1}$ into the unique edge $\psi E$ in $\bar{M}$ with starting point $\psi P$ and label $y$. Moreover, this map $\psi$ can be extended to map the regions of $M$ onto two cells of $\bar{M}$. The map $\psi$ need not be one to one : if $p_{1}, p_{2}$ has the form $r_{1}, r_{1}$, then $M$ consists of two loops bounding disjoint regions at 0 , which doubly cover a loop at $P(1)$ in $\bar{M}$ bounding a single two cell. The possibility of embedding $M$ in the plane, and the consequent multiplicity of $\psi$, results from the fact that, in constructing $M$, we have not taken into account all coincidences between parts of the $p_{i}^{ \pm 1}$, but only those that play a role in cancellations leading to the reduced form of the product $w=p_{1} \ldots p_{n}$.

\section{Free groups}

We now suppose that $F$ is a free group, $R$ a set of non trivial elements of $F$, and $N$ the normal closure of $R$ in $F$. If $w$ is in $N$, then $w$ can be written as a product $w=p_{1} \ldots p_{n}$, for some $n \geqq 0$, of conjugates $p_{i}$ of elements $r^{ \pm 1}$ for $r$ in $R$; indeed, each $p_{i}=u_{i} r_{i} u_{i}^{-1}$ reduced, where each $r_{i}$ is a cyclically reduced conjugate of some $r$ in $R$. Moreover, we can suppose the sequence $p_{1}, \ldots, p_{n}$ reduced. If $M$ is a reduced diagram for this sequence, we shall impose conditions on $R$ which imply conditions on the degrees of interior regions and vertices of $M$, and hence, by our lemmas on maps, imply conditions on $M$.

First, it is convenient to suppose $R$ symmetrized in the sense that every element $r$ of $R$ is cyclically reduced and that, if $r$ is in $R$, then every cyclically reduced conjugate of $r$ or $r^{-1}$ is in $R$. Second, we can without loss delete from 
$M$ every vertex of degree 2 , except possibly 0 , with the result that $d(P) \geqq 3$ for each interior vertex $P$.

Define a piece to be an element $x$ of $F$ such that $R$ contains two distinct elements with reduced forms $r=x a$ and $s=x b$. Suppose that $E$ is an interior edge of $M$, separating regions $D_{1}$ and $D_{2}$. If $x$ is the label on $E$, then $D_{1}^{*}$ and $D_{2}^{*}$ will have labels, starting at the starting point of $E$, which are elements of $R$ of reduced forms $r_{1}=x a$ and $r_{2}=b x^{-1}$. Since the label on $D$; for $D=D_{1} \cup D_{2} \cup E$, is $a b$, and $M$ is reduced, $a b \neq 1$, and $R$ contains distinct elements with reduced forms $x a$ and $x b^{-1}$. It follows that every label on an interior edge is a piece.

If $D$ is an interior region of $M$, and $d(D)=k$, then $D^{\prime}$ is described by an arc $E_{1}, \ldots, E_{k}$ where each $E_{i}$ is an interior edge, and $D^{\circ}$ has a label $r$ in $R$ with reduced form $r=x_{1} \ldots x_{k}$, where each $x_{i}$ is a piece. Consequently the condition that $d(D) \geqq p$ for every interior region $D$ will follow from the following condition on $R$ :

$C(p): \quad$ No element of $R$ is a product of fewer than $p$ pieces.

Case $i$. Assume that $R$ satisfies the condition $C(6)$. Then $d(D) \geqq 6$ for each interior region $D$. Moreover, $d(P) \geqq 3$ for each interior vertex. Suppose $w \neq 1$, whence $M$ contains a critical subdiagram $M_{0}$ satisfying the same conditions on interior degrees. If $M_{0}$ consists of a single region $D$, then some simple closed arc $\delta$ bounding $D$ is a subarc of the arc $\alpha$ describing $M^{*}$ at 0 , whence the label $r$ in $R$ on $\delta$ is a part of the reduced form of the label $w$ on $\alpha$. If $M_{0}$ contains more than one region, then, by Corollary 2.3 , it contains at least two boundary regions $D$ with at most 3 interior edges each. Taking one of these $D$ distinct from the exceptional region $D_{0}$, we have that $D^{\circ} \cap M_{0}$ is a simple arc $\delta$, part of $\alpha$. Now $D^{*}$ is described by an arc $\delta E_{1} \ldots E_{k}$, where $k \leqq 3$ and the $E_{i}$ are interior edges. Then $R$ contains an element $r$ with reduced form $r=a x_{1} \ldots x_{k}, k \leqq 3$, where $a$ is the label on $\delta$ and $x_{1}, \ldots, x_{k}$ are pieces. Moreover, $a$ is a part of the reduced word $w$. In this sense, $w$ contains some $r$ from $R$ with (at most) 3 pieces missing. (We can delete 'at most', since 1 is always a piece.)

Case ii. Assume $C(4)$, whence $d(D) \geqq 4$ of each interior region $D$. If we can ensure that $d(P) \geqq 4$ for each interior vertex $P$, then we can conclude, exactly as in Case $i$, that each non trivial element $w$ in $N$ contains some element $r$ from $R$ with two pieces missing. It remains to formulate a condition on $R$ excluding interior vertices of degree 3 . Suppose that $P$ were such a vertex, with $E_{1}, D_{1}, E_{2}, D_{2}, E_{3}, D_{3}$ in order the edges leading into $P$ and the included regions. The boundary of $D_{i}$, starting at $P$, then has label with reduced form $r_{i}=x_{i}^{-1} a_{i} x_{i+1}$ in $R$, where $i$ is taken modulo 3 and $x_{i}$ is the label on $E_{i}$. Thus there is cancellation in each of the products $r_{1} r_{2}, r_{2} r_{3}$, and $r_{3} r_{1}$. To exclude such vertices we impose on $R$ the following condition:

$T_{3}$ : If $r_{1}, r_{2}$, and $r_{3}$ are in $R$, then in at least one of the products $r_{1} r_{2}, r_{2} r_{3}$, $r_{3} r_{1}$ there is no cancellation.

Case iii. This case is of very limited interest, since the hypotheses needed for our argument seem both artificial and very restrictive; we know of no 
natural application, although we shall give later a natural, if special, application of the analogous case for free products. In fact, the condition $C(3)$ is very mild; it can be given the simpler form that, if $r_{1}, r_{2}$, and $r_{3}$ are elements of $R$, with $r_{1} r_{2} \neq 1$ and $r_{2} r_{3} \neq 1$, then $r_{2}$ does not cancel entirely in the product $r_{1} r_{2} r_{3}$. The condition that all interior vertices have degree $d(P) \geqq 6$ is, on the other hand, very strong. We can ensure this by assuming $T_{3}$ together with analogous $T_{4}$ and $T_{5}$. In fact, since $M$ is reduced, we can modify each of these conditions $T_{k}$ to $T_{k}^{\prime}$ by inserting the additional hypothesis that no $r_{i} r_{i+1}=1$. It is easy to see that $T_{3}^{\prime}$ is in fact equivalent to $T_{3}$, and that, in the presence of $T_{3}, T_{5}^{\prime}$ is equivalent to $T_{5}$. However $T_{4}^{\prime}$ is significantly weaker than $T_{4}$. Even so, if each $r$ in $R$ has length at least $4, T_{4}^{\prime}$ cannot be realized in any case that does not already fall under Case ii. The best we can do to improve this is to replace $T_{4}$ by the following:

$T_{4}^{\prime \prime}: \quad$ If $r_{1}, \ldots, r_{5}$ are elements of $R$, with each $r_{i} r_{i+1} \neq 1$, then there exists $a$ in $F$ such that each $r_{i}^{\prime}=a r a^{-1}$ is in $R$, and in at least one of the products $r_{i}^{\prime} r_{i+1}^{\prime}$ there is no cancellation.

For, it can be shown by an argument similar to that we shall use in the next section, if $M$ contains an interior vertex $P$ of degree 4, assuming $T_{4}^{\prime \prime}$ we can modify $M$ to eliminate this vertex. In all, under hypotheses $C(3), T_{3}, T_{4}^{\prime \prime}$, and $T_{5}$, we can conclude as before that each non trivial element of $N$ contains some $r$ in $R$ with two pieces missing.

We have proved the following theorem.

Theorem I. Let $F$ be a free group, $R$ a symmetrized subset of $F, N$ the normal closure of $R$ in $F$, and $w$ a non trivial element of $N$. Under the additional hypothesis

(i)

$C(6)$,

it follows that $w$ contains some $r$ from $R$ with three pieces missing: $w$ and $r$ have reduced forms $w=$ bac and $r=a x_{1} x_{2} x_{3}$, where $x_{1}, x_{2}$, and $x_{3}$ are pieces.

Under either of the hypotheses

C(4) and $T_{3}$,

or

(iii)

$$
C(3), \quad T_{3}, \quad T_{4}^{\prime \prime}, \quad \text { and } T_{5} \text {, }
$$

it follows that $w$ contains some $r$ from $R$ with two pieces missing.

With each positive real number $\lambda$ we associate the following condition:

$C^{\prime}(\lambda)$ : If $r$ in $R$ has reduced form $r=a b$ where $a$ is a piece, then a has length $|a|<\lambda|r|$.

If $\lambda \leqq \frac{1}{p-1}$, then $r$ cannot be the product of as few as $p-1$ pieces, and $C(p)$ holds. Thus Theorem I has the following corollary.

Corollary 4.1. Let $F, R, N$, and $w$ be as before. If we have

$$
C^{\prime}(\lambda) \quad \text { for some } \lambda \leqq \frac{1}{5},
$$

then $w$ contains a part a of some $r$ in $R$, with $|a|>(1-3 \lambda)|r|$. If we have either

$$
C^{\prime}(\lambda) \text {, for some } \lambda \leqq \frac{1}{3} \text {, and } T_{3} \text {, }
$$


or

(iii) $C^{\prime}(\lambda)$, for some $\lambda \leqq \frac{1}{2}$, and $T_{3}, T_{4}^{\prime \prime}$, and $T_{5}$,

then $w$ contains a part a of some $r$ in $R$, with $|a|>(1-2 \lambda)|r|$.

Most of these results are known. Both ScHiEK [18] and GREENDLINGER [10] have stated results in terms of pieces missing. Case i of the corollary, for $\lambda=1 / 6$, was stated explicitly by GREENDLINGER [9]; earlier TARTAKOvSKII [22] had obtained a closely related result, and BRITTON [3] had obtained the analogous result with the free group $F$ replaced by a free product. It is mentioned in a review by BOONE [2] that BRITTON had conjectured Case $\mathrm{i}$ with $\lambda=1 / 5$, and BRITTON now has a proof by his methods for this case. Case ii, for $\lambda=1 / 4$, was stated explicitly by GREENDLINGER [11]; earlier a related result had been given by SCHIEK [18], who introduced the condition $T_{3}$, and BRITTON [3] appears to allude to a similar result.

DEHN's argument gives an immediate solution of the Word Problem for $R$ finite whenever every non trivial element of $N$ contains more than half of some element from $R$, in particular, under Case i of the corollary provided $\lambda \leqq 1 / 6$, or Case ii provided $\lambda \leqq 1 / 4$. Under a more general hypothesis, a different argument is needed.

Theorem II. Let $F$ be a free group, $R$ a finite symmetrized subset of $F$, and $N$ the normal closure of $R$ in $F$. Assume one of the three hypotheses: $C(6)$,

Then there exists an algorithm for deciding whether arbitrary $w$ in $F$ belongs to $N$.

We show first that if $w$ is in $N$, hence $w=p_{1} \ldots p_{n}$ for some $n \geqq 0$ and $p_{i}=u_{i} r_{i} u_{i}^{-1}$ reduced, $r_{i}$ in $R$, then some such sequence $p_{1}, \ldots, p_{n}$ has a diagram $M$ with no more than $p^{2}|w|^{2}$ vertices, where $p=6,4,3$ according to the case. Indeed, let $p_{1}, \ldots, p_{n}$ be a reduced such that $p_{1} \ldots p_{n}=w$, and let $M$ be the associated reduced diagram. If a closed arc $\alpha$ at 0 describing $M$ has $k$ edges, then $w$ is the product without cancellation of the $k$ non trivial labels on these edges, whence $k \leqq|w|$. Each vertex on $M$ occurs at least once as the starting vertex of some edge in $\alpha$, whence the number $a^{\circ}$ of vertices on $M$ is no greater than $k$, that is, $a^{-} \leqq k$. The length of $M$, in the sense of Lemma 2.6 , is given by $b=\Sigma[p-d(P)]$, whence it follows that $b=\Sigma[p-d(P)] \leqq \Sigma p=p a^{\circ} \leqq p k \leqq p|w|$. Now Lemma 2.6 gives $a \leqq b^{2} \leqq p^{2}|w|^{2}$.

Now suppose arbitrary $w$ in $F$ given. If $w$ is in $N$, it must be the product of a sequence with a reduced diagram with no more than $p^{2}|w|^{2}$ vertices. There are, within isomorphism, only finitely many unlabeled maps with no more than this many vertices, whence it will suffice, given $w$ in $F$, to decide whether a given such map can be labeled to yield a diagram of the required sort. Now a label on an interior edge is a piece, and, since $R$ is finite, there are only finitely many pieces. A label on a boundary edge must be part of the reduced word $w$, 
and there are only finitely many such parts. Thus, given a map, there are only finitely many labelings of its edges to consider, and it suffices to decide whether a given labeled map in fact constitutes a diagram for a sequence $p_{1}, \ldots, p_{n}$ of conjugates of elements $r_{i}$ in $R$, with $p_{1} \ldots p_{n}=w$. But this last equation can clearly be settled by inspection.

We state now the last of our three main results.

Theorem III. Let $F$ be a free group, $R$ a symmetrized subset of $F$, and $N$ the normal closure of $R$ in $F$. The operation of $F$ on $N$ by conjugation induces on the quotient group $N_{0}=N /[N, N]$ of $N$ by its derived group the structure of an $F / N$ module. Assume one of the following three hypotheses:

$$
\begin{aligned}
& C(6), \\
& C(4) \text { and } T_{3},
\end{aligned}
$$

Then the $F / N$ module $N_{0}$ is the direct sum of cyclic submodules.

For each $r$ in $R$, the coset $r[N, N]$ in $N_{0}$ generates a cyclic submodule, containing all $p[N, N]$ for $p$ conjugate to $r$; clearly conjugate elements $r$ of $R$ generate the same submodule. We shall show that $N_{0}$ is the direct sum of these cyclic submodules. This amounts to showing that if $w=p_{1} \ldots p_{n}$ is in $[N, N]$, where each $p_{i}$ is a conjugate of some element from $R$, then the factors $p_{i}$ fall into pairs $p_{i}$ and $p_{j}$, with $p_{i}=q p_{j}^{-1} q^{-1}$ for some $q$ in $N$. Since a commutator $p_{i}^{-1} p_{j}^{-1} p_{i} p_{j}$ of conjugates of elements of $R$ obviously has this pairing property, it suffices, by an induction on $n$, to show that if $p_{1} \ldots p_{n}=1$, and $n>0$, then there is one pair of factors $p_{i}$ and $p_{j}$ of the required sort. We shall show more, that $p_{1} \ldots p_{n}=1$ implies the sequence $p_{1}, \ldots, p_{n}$ is not reduced.

Suppose, $w=p_{1} \ldots p_{n}, n>0$, each $p_{i}$ conjugate to some element of $R$, and that the sequence $p_{1}, \ldots, p_{n}$ is reduced. By Lemma 3.3, there exists a reduced diagram $M$ for this sequence, together with some number $q \geqq 0$ of reduced diagrams $L_{1}, \ldots, L_{q}$ on the sphere, containing in all $n$ regions. By Corollary 2.5 , there cannot exist any map $L$ on the sphere satisfying those conditions on degrees that follow from the hypotheses of Theorem II. Therefore $q=0$ and $M$ contains $n$ regions. But then $M$, and so also $M$, consists of more than a single point. It follows that $w \neq 1$.

In the cases where one can conclude that every non trivial element of $N$ contains more than half of some element from $R$, CoHEN and LYNDON [4] derived the conclusion of Theorem IIIfrom a stronger result, that $N$ has a free basis consisting of conjugates of elements from $R$. It is not clear whether this stronger result holds in the more general situation considered in Theorem III.

\section{Free products}

We now suppose that $F$ is no longer a free group, but the free product of a given family of subgroups $F_{i}$, with distinct $F_{i}$ having trivial intersection. We shall again take $R$ to be a subset of $F$ and $N$ the normal closure of $R$ in $F$. The construction of diagrams and the proofs of Theorems I, II, and III then go 
over with very little change. We shall confine ourselves to the proof of an analog of Theorem I, and to giving two simple applications.

Every element $w$ of $F$ now has a unique normal form, $w=w_{1} \ldots w_{n}, n \geqq 0$, where each of the letters $w_{i}$ is a non trivial element of some component $F_{j}$, and adjacent $w_{i}, w_{i+1}$ do not belong to the same $F_{j}$. The length of such $w$ is $|w|=n$. Given two elements with normal forms $u=u_{1} \ldots u_{m}$ and $v=v_{1} \ldots v_{n}$, in obtaining the normal form for the product $w=u v$ there may be cancellation of a certain number of letters from the end of $u$ against their inverses, at the beginning of $v$; it may also happen that the last remaining letter $u_{i}$ of $u$ is in the same $F_{k}$ as the first remaining letter $v_{j}$ of $v$, in which case they are consolidated to give a single letter $u_{i} v_{j}=w_{i}$ in the normal form for $w$. We shall speak of $w$ as having reduced form $w=u v$ if there is no cancellation in forming the product; we do not exclude the possibility of consolidation. It should be emphasized that for $a$ to be a part of $w$ with reduced form $w=b a c$ does not require that the letters of $a$ should be a subsequence of those of $w$.

We shall call an element $r$ cyclically reduced if it has normal form $r=r_{1} \ldots r_{n}$ where $r_{n} r_{1} \neq 1$; if $r_{1}$ and $r_{n}$ belong to different $F_{j}$, we call $r$ strictly cyclically reduced. A set $R$ will be called symmetrized if every $r$ in $R$ is cyclically reduced and if, together with each $r$ in $R$, every cyclically reduced conjugate of $r$ on $r^{-1}$ also belongs to $R$. The definition of a piece is modified as follows: $x$ is a piece if $R$ contains elements with reduced forms $a x b$ and $c x d$ where $x b a \neq x d c$.

We turn now to the construction of a diagram $M$ associated with a sequence $p_{1}, \ldots, p_{n}$. In all diagrams considered, the vertices will fall into two classes, primary and secondary, and the two ends of every edge will fall into different classes. The label on every edge will belong to some $F_{i}$, with the labels on two edges meeting successively at a primary vertex belonging to different $F_{i}$, and those on edges meeting at a secondary vertex belonging to the same $F_{j}$. If $E_{1}, \ldots, E_{k}$ are in order the edges running into a secondary vertex $P$, then we shall want to specify the values of the labels $\phi\left(E_{i}^{-1} E_{i+1}\right)=\phi\left(E_{i}\right)^{-1} \phi\left(E_{i+1}\right)$, but we shall not be concerned with the particular choice of the $\phi\left(E_{i}\right)=x_{i}$. For the $x_{i}$ we shall then have a system of equations $x_{i}^{-1} x_{i+1}=\phi\left(E_{i}^{-1} E_{i+1}\right)=a_{i}$, in $F_{j}$, for $i$ modulo $k$, and of inequations $x_{i} \neq 1$. We shall have always the compatibility condition $a_{1} \ldots a_{k}=1$; with this one of the equations $x_{i}^{-1} x_{i+1} \equiv a_{i}$ becomes redundant, and the rest can be solved to express the remaining $x_{i}$ in terms of $x_{1}$. We are then left with $k$ inequations $x_{1} \neq b_{1}, \ldots, b_{k}$. Assuming, as we may in this context, that each $F_{j}$ is infinite, a solution can always be found.

With a sequence of length $n=0$ we associate as before a diagram $M$ consisting of the single (primary) point $O$. Let $n=1$; then $p_{1}$ has normal form $p_{1}=a_{1} \ldots a_{k} b_{1} \ldots b_{m} a_{k}^{-1} \ldots a_{1}^{-1}$ where $k \geqq 0, m \geqq 1$, and $b_{m} b_{1} \neq 1$. If $b_{1}$ and $b_{m}$ are in different $F_{j}$ we take $M$ to be a loop at $P$ joined to $O$ by a path. The path $O P$ consists of $2 k$ edges $E_{1}, E_{1}^{\prime}, \ldots, E_{k}, E_{k}^{\prime}$, with each $\phi\left(E_{i} E_{i}^{\prime}\right)=a_{i}$. The loop at $P$ consists of $2 m$ edges $B_{1}, B_{1}^{\prime}, \ldots, B_{m}, B_{m}^{\prime}$, with each $\phi\left(B_{i} B_{i}^{\prime}\right)=b_{i}$. If $b_{1}$ and $b_{2}$ lie in the same $F_{j}$, we take $O P$ to end with an additional edge $E$, and the loop at $P$ to consist of edges $B, B_{2}, B_{2}^{\prime}, \ldots, B_{m-1}, B_{m-1}^{\prime}, C$, with $\phi\left(B_{i} B_{i}^{\prime}\right)=b_{i}$ as before for $2 \leqq i \leqq m-1$. The three edges $E, B$, and $C$ at the secondary vertex $P$ are to be 
labeled in such a way as to satisfy the compatible conditions $\phi(E B)=a_{1}$, $\phi\left(C E^{-1}\right)=a_{m}$ and $\phi(C B)=a_{m} a_{1}$.

The argument by induction for $n>1$ follows the same plan as before. Suppose at some stage we have successive edges $E, E^{\prime}, G$, and $G^{\prime}$, where $E$ and $G$ are separated by a primary vertex $P$, and yet $\phi\left(E E^{\prime}\right)=a$ and $\phi\left(G G^{\prime}\right)=b$ lie in the same $F_{j}$. If $a b=1$, we either delete a loop bounded by these four edges, or else, as before, we bring $E^{\prime}$ into coincidence with $G^{-1}$ (their secondary vertices cannot coincide) and $E$ with $G^{\prime-1}$. If $a b \neq 1$, we bring $E^{\prime}$ into coincidence with $G^{-1}$, leaving on the boundary consecutive edges to be labeled $\phi\left(E G^{\prime}\right)=a b$. In either case it is necessary, but possible, to readjust the labels on the four edges involved.

With this, we obtain analogs of everything in Section 3.

To begin with an analog. Theorem IV, of Theorem I, we again assume a condition $C(p)$ to ensure that every interior region $D$ of a reduced diagram $M$ has degree $d(D) \geqq p$. As before, we can also suppose that every interior vertex $P$ has degree $d(P) \geqq 3$. For the case that $p=6$, no more is required.

In Case ii, assuming $C(4)$, we again need a condition to exclude interior vertices of degree 4 . To exclude such primary vertices, we require the following variant of $T_{3}$ :

$T_{3}^{1}$ : If $r_{1}, r_{2}$, and $r_{3}$ are strictly cyclically reduced elements of $R$, then, in at least one of the products $r_{1} r_{2}, r_{2} r_{3}, r_{3} r_{1}$, there is non cancellation.

At a secondary vertex $P$ of degree 3 , with successive edges $E_{1}, E_{2}$, and $E_{3}$ leading into $P$, let $x_{1}, x_{2}, x_{3}$ be the labels on these edges. Then the three regions $D_{i}$ meeting at $P$ will bear on their boundaries labels $r_{i}$ in $R$, strictly cyclically reduced, where $r_{i}$ contains a letter $a_{i}=x_{i+1} x_{i}^{-1}$. Thus $a_{3} a_{2} a_{1}=1$. To exclude this, we require the following condition:

$T_{3}^{2}$ : If $a_{1}, a_{2}$, and $a_{3}$ are letters occurring in strictly cyclically reduced elements of $R$, then $a_{1} a_{2} a_{3} \neq 1$.

This suffices for the case $p=4$.

The remaining Case iii is again awkward. We again assume $C(3)$, and also $T_{3}^{1}, T_{4}^{1}$, and $T_{5}^{1}$, obtained from $T_{3}, T_{4}^{\prime \prime}$, and $T_{5}$ by restricting consideration to strictly cyclically reduced $r_{i}$ in $R$. These imply the condition $d(D) \geqq 3$ on interior regions $D$, and the condition $d(P) \geqq 6$ on interior primary vertices. For interior secondary vertices, $T_{3}^{2}$ together with analogous $T_{4}^{2}$ and $T_{5}^{2}$ would suffice, but are needlessly strong. If we restrict these conditions to the case where all $a_{i} a_{i+1} \neq 1$, the content of $T_{3}^{2}$ and $T_{5}^{2}$ taken together is not altered, but we obtain $T_{4}^{2}$ in weaker form:

$T_{4}^{2}$ : If $a_{1}, a_{2}, a_{3}, a_{4}$ are letters occuring in strictly cyclically reduced elements of $R$, and $a_{1} a_{2}, a_{2} a_{3}, a_{3} a_{4} \neq 1$, then $a_{1} a_{2} a_{3} a_{4} \neq 1$.

This condition does not in fact exclude that the diagram $M$ should contain an interior secondary vertex $P$ of degree $d(P)=4$, but does ensure that $M$ can be modified in such a way as to eliminate all such vertices. Suppose then that $P$ is such a vertex, with four edges $E_{i}=P_{i} P$ running into $P$, in order, and with 
regions $D_{i}$ lying between $E_{i}$ and $E_{i+1}$, where $i$ is taken modulo 4 . Since $P$ is secondary, the labels $x_{i}=\phi\left(E_{i}\right)$ all lie in the same $F_{j}$, and the $a_{i}=x_{i+1} x_{i}^{-1}$ satisfy $a_{4} a_{3} a_{2} a_{1}=1$. By $T_{4}^{2}$ and symmetry we can suppose $a_{2} a_{1}=1$, hence $x_{1}=x_{3}$. In the diagram $K=D_{1} \cup \cdots \cup D_{4}$, we think of the pair of edges $E_{1}=P_{1} P$ and $E_{3}^{-1}=P P_{3}$ as obtained by flattening a rhombus $P_{1} P^{2} P_{3} P^{4}$ onto a diagonal in such a way that $P^{2}=P^{4}=P$. We obtain $K^{\prime}$ by flattening this rhombus onto its other diagonal, so that $P_{1}$ and $P_{3}$ coincide at a point $Q$. Since $K^{\prime}$ has the same boundary as $K$, we can form $M^{\prime}$ from $M$ by replacing the part $K$ by $K^{\prime}$. Now $M^{\prime}$ does not contain the vertex $P$, but contains three vertices that did not appear in $M$. Two of these, $P^{2}$ and $P^{4}$, are of degree 2 , and can be deleted, while the third, $Q$, is a primary vertex. Since $M^{\prime}$ contains fewer interior vertices of degree 4 than $M$, by iteration of this process we can eliminate all such vertices.

With this, we have proved an analog of Theorem I.

Theorem IV. Let $F$ be a free product, $R$ a symmetrized subset of $F$, and $w$, a non trivial element in the normal closure $N$ of $R$ in $F$. Assuming

we conclude that $w=$ bac reduced, where $r=a x_{1} x_{2} x_{3}$ reduced, for some $r$ in $R$ and pieces $x_{1}, x_{2}, x_{3}$.

Assuming either

$C(4), T_{3}^{1}$ and $T_{3}^{2}$,

or

$$
C(3) \text { and } T_{i}^{1}, T_{i}^{2}, i=3,4,5 \text {, }
$$

we conclude that $w=$ bac reduced, where $r=a x_{1} x_{2}$ reduced, for some $r$ in $R$ and pieces $x_{1}$ and $x_{2}$.

If we replace the hypothesis $C(p)$ by $C^{\prime}(\lambda)$, for $\lambda<\frac{1}{p-1}$, then the exact analog of Corollary 4.1 holds, with the conclusion that $|a|>(1-3 \lambda)|r|$ or $|a|>(1-2 \lambda)|r|$ according to the case. This is not quite immediate since, for example, we may have $\left|x_{1} x_{2} x_{3}\right|<\left|x_{1}\right|+\left|x_{2}\right|+\left|x_{3}\right|$ if there is consolidation in forming the product. However, it is easy to see that corresponding considerations strengthen the hypothesis $C^{\prime}(\lambda)$ in a way that more than compensates for this.

It should be emphasized that, in Theorem IV, the part $a$ of $w$ need not consist precisely of a consecutive sequence of factors from the normal form of $w$, or of $r$. If we require this, and also restrict a piece to be a consecutive part in this sense of the normal forms of two distinct strictly cyclically reduced elements of $R$, then, for the reasons indicated, we can assure only that $|a|>(1-3 \lambda)|r|-4$ or $|a|>(1-2 \lambda)|r|-3$, according to the case. A procedure that is in some sense more natural is to replace $|a|$, as part of $w=b a c$, by the number of full factors from $w$ in $a$, and adding $1 / 2$ for a factor on either end of $a$ that is consolidated in forming $w$; if this is done consistently, the number $\|a\|$ exceeds $(1-3 \lambda)|r|$ or $(1-2 \lambda)|r|, r$ strictly cyclically reduced. This is similar to the procedure of BRITTON [3]. 
We conclude with two examples. The case with $C(6)$, in particular with $\lambda \leqq 1 / 6$, either for $F$ a free group or $F$ a free product, has already been adequately illustrated by DEHN and his successors. We shall illustrate the two remaining cases, for $F$ a free product. It must be confessed that our example for the third case is the only reasonable application of this case that we have been able to discover.

Example 5.1. Let $F=A * B$, free product of subgroups $A$ and $B$ with $A \cap B=1$. Let $r=a_{1} b_{1} a_{2} b_{2}$ with $a_{1}, a_{2}$ in $A$ and $b_{1}, b_{2}$ in $B$, and let $N$ be the normal closure of $r$ in $F$. Assume that the set of $a_{i}^{ \pm 1}$ and $b_{i}^{ \pm 1}$ consists of eight distinct elements, and that no one is the square of another. Then $N \cap A=N \cap B=1$.

We take $R$ to consist of all cyclically reduced conjugates of $r$ and $r^{-1}$. If $s$ is any strictly cyclically reduced element of $R$, it follows from the assumption that the $a_{i}^{ \pm 1}$ and $b_{i}^{ \pm 1}$ are distinct, that a piece of $s$ cannot contain all of any letter from $s$, and hence consists at most of parts of two adjoining letters. Thus $s$ cannot be a product of three pieces, and $C(4)$ holds. Condition $T_{3}^{1}$ is immediate on the same grounds: there cannot be cancellation in the product st of two strictly cyclically reduced elements of $R$ unless $s t=1$. For $T_{3}^{2}$ we require that no product of three factors $a_{i}^{ \pm 1}$ or $b_{i}^{ \pm 1}$ is trivial, and this has been incorporated in our hypothesis. It follows now that if $w$ is any non trivial element of $N$, then $w=b a c$ reduced, where some $s=a x_{1} x_{2}$, reduced, $s$ in $R$ and $x_{1}, x_{2}$ pieces. Evidently the part $a$ of $s$ cannot lie in $A$ or in $B$, whence the same holds for $w$.

Example 5.2. Let $F=A * B$, free product of subgroups $A$ and $B$ with $A \cap B=1$. Let $r=a_{1} b_{1} a_{2} b_{2} a_{3} b_{3}$ with the $a_{i}$ in $A$ and the $b_{i}$ in $B$, and let $N$ be the normal closure of $r$ in $F$. Assume that the $b_{i}$ are strictly 5-free in the following sense: if $b_{i_{1}}^{e_{1}} \ldots b_{i_{m}}^{e_{m}}=1$, where $1 \leqq m \leqq 5$, each $i_{h}=1,2$, or 3 , and each $e_{h}= \pm 1$, then there exists $h, 1 \leqq h<m$, such that $i_{h}=i_{h+1}$ and $e_{h}=-e_{h+1}$. Assume that the $a_{i}$ are weakly 5-free in the following sense: if $a_{i_{1}}^{e_{1}} \ldots a_{i_{m}}^{e_{m}}=1 ; 1 \leqq m \leqq 5$; $i_{h}=1,2$, or $3 ; e_{h}= \pm 1$; then $a_{i_{h}}^{e_{h}} a_{i_{h+1}}^{e_{h+1}}=1$ for some $h, 1 \leqq h<m$. Then $N \cap A$ $=N \cap B=1$.

Take $R$ as before. It follows from the distinctness of the $b_{i}^{ \pm 1}$ that no piece can contain a letter $b_{i}^{ \pm 1}$ except as its first or last letter, and hence cannot contain two letters $a_{i}^{ \pm 1}$. Consequently no element of $R$ is a product of two pieces, and $C(3)$ holds. (We note that $C(4)$ need not hold, for example if $a_{1}=a_{2}=a_{3}$.) Again the distinctness of the $b_{i}^{ \pm 1}$ implies that one cannot have strictly cyclically reduced $r_{i}$ in $R$ such that for some $i$ there is cancellation in both $r_{i-1} r_{i}$ and $r_{i} r_{i+1}$, with neither product trivial; thus $T_{3}^{1}, T_{4}^{1}$, and $T_{5}^{1}$ hold. The weak 5 -freeness of the letters gives precisely $T_{3}^{2}, T_{4}^{2}$, and $T_{5}^{2}$. The conclusion of the theorem, that non trivial $w$ in $N$ contains some element from $R$ with two pieces missing is here not strong enough. For let $a_{1}=a_{2}=a_{3}=a$. Then $x_{1} \equiv b_{1} a b_{2}$ begins both $b_{1} a b_{2} a b_{3} a$ and $b_{1} a b_{1} a b_{2} a\left(b_{3} b_{1}^{-1}\right), x_{2}=a b_{3}$ begins both $a b_{3} a b_{1} a b_{2}$ and $a b_{1} a b_{2} a b_{3}$, and $r=a x_{1} x_{2}$. We appeal to the diagram $M$, which we know to contain a region $D$ with at most two interior edges, restoring to $D^{\prime}$ its three primary vertices and its three secondary vertices, dividing $D^{\prime}$ into what we shall call subedges. No interior edge can contain two successive subedges with labels in $B$, by virtue of the distinctness of the $b_{i}^{ \pm 1}$. Therefore an edge can at most 
contain two adjacent subedges with labels in $A$, together with a subedge with label in $B$ at each end. Two such edges can exhaust at most eight of the twelve subedges of $D^{*}$, whence the part $D^{*} \cap M^{\prime}$ must contain at least four subedges, and hence bear a label that does not lie in $A$ or in $B$. We conclude again that $w$ is not in $A$ or $B$.

Corollary 5.3. Let $B$ be any group, and $b_{1}, b_{2}$, and $b_{3}$ three elements of $B$ that are 5-free in the above sense. Then $B$ can be embedded in a group $G$ containing an element $x$ such that, in $G, x b_{1} x b_{2} x^{-1} b_{3}=1$.

Let $A$ be a cyclic group, with generator $a$, of order $n \neq 1,2,3,4,5$. Let $F=A * B$ and $r=a b_{1} a b_{2} a^{-1} b_{3}$, with $N$ the normal closure of $R$ in $F$. The hypothese of Example 5.2 are satisfied, and we conclude that $N \cap B=1$. Consequently $G=F / N$ contains $B$ isomorphically, and the element $x=a N$ satisfied the required equation. We note that $B$ is contained in no proper normal subgroup of $G$, for, if $K$ is the normal closure of $B$ in $G$, then $G / K$ is generated by $x K$; with a defining relation implying that $x K=1$.

We remark that the above corollary gives only a rather special example of a variety of similar results that can be obtained by similar methods. A stronger form of the result given is contained in a theorem of GERSTENHABER and RoTHAus [7] under the assumption that $B$ can be embedded in a compact connected Lie group. The analogous result for the equation $x b_{1} x b_{2} x b_{3}=1$ follows from a simpler argument of LEVIN [13]. If one considers more generally an equation $x^{n_{1}} b_{1} \ldots x^{n_{k}} b_{k}=1$, the case $k=1$ is trivial, while partial results for $k=2$ and $k=3$ can be derived from the two examples above. For $k=4$ or $k=5$, Case ii of Theorem IV can be applied under somewhat milder conditions on the $n_{i}$ and $b_{i}$. For $k \geqq 6$, we can use Case i of Theorem IV, and conclude that a solution exists provided all $n_{i} \neq 0$ and all $b_{i}^{ \pm 1}$ are non trivial and distinct; in particular, one may well have $\Sigma n_{i}=0$.

\section{Bibliography}

[1] Berge, C.: Théorie des graphes et ses applications. Paris: Dunod 1958.

[2] Boone, W. W.: Review of Britron [3]. Math. Reviews 23, 440-441 (1962), A 2325.

[3] BRITTON, J. L.: Solution of the word problem for certain types of groups, I, II. Proc. Glasgow Math. Ass. 3, 45-54, 68-90 (1956-58).

[4] CoHen, D. E., and R. C. LYNDON: Free bases for normal subgroups of free groups. Trans. Am. Math. Soc. 108, 528-537 (1963).

[5] DeHN, M.: Uber unendliche diskontinuierliche Gruppen. Math. Ann. 71, 116-144 (1912).

[6] - Transformation der Kurven auf zweiseitigen Flächen. Math. Ann. 72, 413-421 (1912).

[7] Gerstenhaber, M., and O. S. Rothaus: The solution of sets of equations in groups. Proc. Nat. Acad. Sci. U.S. 48, 1531-1533 (1962).

[8] GlaDKII, A. V.: On simple Dyck words. Sibirsk Mat. Zh. 2, 36-45 (1961).

[9] Greendlinger, M.: Dehn's algorithm for the word problem. Comm. Pure Appl. Math. 13, $67-83(1960)$.

[10] - On Dehn's algorithms for the conjugacy and word problems with applications. Comm. Pure Appl. Math. 13, 641-677 (1960).

[11] - Solutions of the word problem for a class of groups by means of Dehn's algorithm, and of the conjugacy problem by means of a generalization of Dehn's algorithm. Dokl. Akad. Nauk SSSR 154, 507-509 (1964). 
[12] - Solution of the conjugacy problem for a class of groups coinciding with their anti-centers, by means of the generalized Dehn algorithm. Dokl. Akad. Nauk SSSR 158, 1254-1256 (1964).

[13] LEvin, F.: Solutions of equations over groups. Bull. Am. Math. Soc. 68, 603-604 (1962).

[14] LipschuTZ, S.: Elements in $S$-groups with trivial centralizers. Comm. Pure Appl. Math. 13, $679-683(1960)$.

[15] Lyndon, R. C.: Cohomology theory of groups with a single defining relation. Ann. of Math. $52,650-665$ (1950).

[16] - Dependence and independence in free groups. Crelles J. 210, 148-174 (1962).

[17] REIDEMEISTER, K.: Einführung in die kombinatorische Topologie. Braunschweig: Vieweg 1932.

[18] Schiex, H.: Ähnlichkeitsanalyse von Gruppenrelationen. Acta Math. 96, 157-251 (1956).

[19] - Das Adjunktionsproblem der Gruppentheorie. Math. Ann. 147, 159-165 (1962).

[20] TARTaKovskil, V. A.: The sieve method in group theory. Mat. Sbornik (N.S.) 25, (67), 3-50 (1949).

[21] - Application of the sieve method to the solution of the word problem for certain types of groups. Mat. Sbornik (N.S.) 25, (67), 251-274 (1949).

[22] - Solution of the word problem for groups with a $k$-reduced basis for $k>6$. Izvestiya Akad. Nauk SSSR, Ser. Mat. 13, 483-494 (1949).

[23] - On primitive composition. Mat. Sbornik (N.S.) 30, (72), 39-52 (1952).

[24] - Translations of $[20,21,22]$. Am. Math. Soc. Translations 60 (1952), reprint 1 (1962).

[25] Zieschang, H.: Studien zur kombinatorischen Topologie von Flächen und ebenen diskontinuierlichen Gruppen (multigraphed). Frankfurt 1964 (priv. Veröffentlichung).

Added in proof. We would like to add to the bibliography several references that have come recently to our attention. First, independent work of C. WeinBaum [34] appears to have much in common with the present paper; also, WEINBAuM drew our attention to a paper of E. VAN KAMPEN [33] containing essentially our concept of diagram. We are grateful to B. GRüNBAuM for telling us of the papers $[26,27]$ of C. BLANC, and the paper [28] of F. FiaLA, where geometric theorems for graphs that are similar to ours are used for a different purpose. We are grateful to M. GREENDLINGER for reference to the papers $[29,30]$ of A. V. GLADKIn, where different proofs of some of our results may be found. We wish also to draw attention to the thesis [32] of P. E. ScHUPp, in which the present methods are extended to apply to the problems of conjugacy, of centralizers, and of torsion, and also to the thesis [31] of P. P. N. ORLiK, written under the guidance of F. RAYMOND, in which these ideas are applied to the theory of manifolds.

Additional bibliography

[26] BlanC, C.: Une interprétation élémentaire des théorèmes fondamentaux de M. Nevanlinna. Comm. Math. Helv. 12, $153-163$ (1940).

[27] - Les résaux Riemanniens. Comm. Math. Helv. 13, $54-67$ (1941).

[28] Fiala, F.: Sur les polyèdres à faces triangulaires. Comm. Math. Helv. 19, 83-90 (1946).

[29] GLADKIl, A. V.: On groups with $k$-reducible bases. Dokl. Akad. Nauk SSSR 134, $16-18$ (1960).

[30] - On groups with $k$-reducible bases. Sibirsk Math. J. 2, 366-383 (1961).

[31] OrLIK, P.P. N.: Necessary conditions for the homeomorphism of Seifert-manifolds. Thesis, University of Michigan, 1966.

[32] Schupp, P. E.: On Dehn's algorithm and the conjugacy problem. Thesis, University of Michigan, 1966. (To be submitted to Math. Ann.)

[33] VAN KAMPEN, E. R.: One some lemmas in the theory of groups. Ann. J. Math. 55, 268-273 (1933).

[34] Weinbaum, C. M.: Visualizing the word problem, with an application to sixth groups. Pacific J. Math. 16, 557-578 (1966).

ROGER C. LYNDON

The University of Michigan, Department of Mathematics, Angell Hall Ann Arbor, Michigan, USA 